\title{
Can Liquid Biopsy be Used to Explore Future Precision Medicine for Solid Tumors?
}

\author{
Hirotoshi Kikuchi, MD, PhD, and Hiroya Takeuchi, MD, PhD \\ Department of Surgery, Hamamatsu University School of Medicine, Hamamatsu, Japan
}

Although the concept of precision medicine has been recognized as a useful tool in health care, its clinical application for patients with solid tumors has remained limited for many years. In recent years, precision medicine has become popular as next-generation sequencing (NGS) technology has progressed and enabled comprehensive genomic profiling (CGP) by decreasing its cost. ${ }^{1}$

Generally, CGP by NGS is performed using tissue specimens obtained by surgical resection or biopsy specimens. When NGS analysis is performed using primary tumor tissues obtained during previous surgery, the results show the previous status of genomic profiling in the primary tumor, but they do not necessarily reflect the current status of recurrent tumors after their development of resistance to standard therapies. Moreover, collecting biopsy specimens from recurrent tumors often is difficult because of their invasiveness. Therefore, liquid biopsy is the preferred option for real-time sample collection for NGS. Liquid biopsy can be performed repeatedly in a minimally invasive or noninvasive manner, even for cancers associated with difficulty in biopsy sampling. ${ }^{2}$

Liquid biopsies can examine either tumor cells or tumor cell products (cell free). ${ }^{3}$ Analysis of circulating tumor cells (CTCs) is a major cell-based liquid biopsy method that can be a powerful tool for biologic analyses of solid tumors. However, it is difficult to collect CTCs because of their rarity in the blood. ${ }^{4}$ In contrast, cell-free liquid biopsy is recognized as an easier method for comprehensive

(C) Society of Surgical Oncology 2021

First Received: 9 March 2021

Accepted: 20 September 2021;

Published Online: 9 October 2021

H. Takeuchi, MD, PhD

e-mail: takeuchi@hama-med.ac.jp profiling of gene mutations, mRNA expression, miRNA expression, protein expression, and exosomes. Plasma cellfree DNA (cfDNA) is therefore a major candidate for precision medicine. $^{4}$

In previous studies, liquid biopsy using circulating tumor DNA (ctDNA) was reported to have some advantages over tissue CGP such as a shorter turnaround time and more appropriate late-line treatment of colorectal cancers. ${ }^{5,}{ }^{6}$ In addition, plasma CGP by ctDNA analysis can analyze whole malignant tissues and overcome the critical issue of heterogeneity in tissue CGP. ${ }^{7}$

Although the utility of liquid biopsy using plasma cfDNA for detecting actionable mutations associated with clinical response is reported in some studies performed in the United States, ${ }^{8,9}$ it has remained unclear whether these results can be applied in other countries with less accessibility to off-label therapy, including Japan.

A recent study compared the trial enrollment rates between two studies performed in the same centers in Japan for advanced gastrointestinal (GI) cancers: SCRUMJapan GOZILA, an observational ctDNA-based screening study, and GI-SCREEN, a tumor tissue-based sequencing study. ${ }^{10}$ Compared with tissue genotyping, ctDNA genotyping shortened the screening duration (11 vs. 33 days) and increased the trial enrollment rate $(9.5 \%$ vs. $4.1 \%)$ without compromising treatment efficacy. ${ }^{10}$ Although this study showed the usefulness of ctDNA for plasma CGP in routine patient care by comparing ctDNA- and tissue-based genotyping in the same trial environment, the comparisons were performed retrospectively and between different sequencing technology platforms. ${ }^{10}$

Matsudera et al. ${ }^{11}$ reported the results of the Precision Cancer Medicine Registration Study of Omics Data From Genomic Information Analysis Leading to New Effective Therapy (PROFILE), a prospective observational study that investigated the clinical utility of comprehensive 
genomic profiling by liquid biopsy using plasma cfDNA in 102 Japanese patients with advanced solid tumors either refractory or likely refractory to standard therapies. Actionable mutations were discovered in $88(86.3 \%)$ of the 102 patients.

Based on the results of the liquid biopsy, 22 of the patients $(21.6 \%)$ received biomarker-matched inhibitors targeting cyclin-dependent kinase 4/6 (CDK4/6), poly(ADP-ribose) polymerase (PARP), epidermal growth factor receptor (EGFR), programmed cell death 1 (PD-1), anaplastic lymphoma kinase (ALK), human epidermal growth factor receptor 2 (ERBB2/HER2), mitogen-activated protein kinase kinase (MEK), fibroblast growth factor receptor (FGFR), phosphatidylinositol-4,5-bisphosphate 3-kinase catalytic subunit alpha (PIK3CA), or insulin-like growth factor 1 (IGF1). The objective response rate was $18.2 \%$, and the disease control rate (DCR) was $50 \%$. Notably, the DCR and median overall survival were significantly better for the patients with a high matching score $(\geq 50 \%)$ than for those with a low matching score $(<$ $50 \%)(75 \%$ vs. $20 \%[P=0.010]$ and 621 vs. 196 days $[P=$ 0.00075], respectively). ${ }^{11}$ This study also showed that the concordance rate between the liquid biopsy and the genomic sequencing results in the primary tumors of 19 patients with GI cancer was 57.9\%. ${ }^{11}$ These results showed the usefulness of liquid biopsy using plasma cfDNA for identifying actionable mutations associated with clinical response for Japanese populations, especially patients with a high matching score of $50 \%$ or higher.

In both the PROFILE study and the SCRUM-Japan GOZILA study, Guardant 360 (Guardant Health, Inc.), a Clinical Laboratory Improvement Aamendments (CLIA)certified, FDA-approved NGS-based liquid biopsy method was used to detect single nucleotide variants (SNVs), deletion variants (indels), fusions, and copy number alterations in 74 genes. $^{10,} 11$ Solid tumors are genetically regulated by SNVs, indels, or amplifications. In addition, epigenetic regulation also is involved in tumor development and progression. ${ }^{8}$ Although Guardant 360 included some epigenetic regulators such as the AT-rich interacting domain containing protein $1 \mathrm{~A}$ gene and enhancer of zeste homolog 2, none of the patients in the PROFILE study received inhibitors targeting these molecules. Some background issues could have been present that needed to be addressed.

The plasma CGP by ctDNA analysis has some limitations and problems, such as the effects from clonal hematopoiesis of indeterminate potential, emphasizing the importance of matched cfDNA white blood cell-sequencing for accurate variant interpretation. ${ }^{12}$ In addition, tissue CGP may be superior to plasma CGP in deciphering some tumors such as brain, bladder, and pancreatic cancers. ${ }^{13,14}$
In Japan, two gene panel tests, FoundationOne CDx Cancer Genomic Profile (Chugai, Tokyo, Japan) and OncoGuide NCC Oncopanel System (Sysmex, Kobe, Japan), that include 324 and 114 genes, respectively, for tissue CGP, currently are approved for reimbursement. Further clinical research should be conducted with nationwide populations to characterize the clinical utility of liquid biopsy compared with the standard tissue CGP for precision medicine in Japanese patients with advanced solid tumors. The concordance rate between tissue- and ctDNA-profiling would be of great interest because it may vary depending on the gene alterations and the interval between the tissue biopsy and the blood draw used for testing. ${ }^{8}$

As the cost of NGS decreases over time, CGP for a large number of cancer biomarkers or even for whole-genome sequencing will be more commonly available. Therefore, liquid biopsy could be performed repeatedly as a major diagnostic tool in future precision medicine.

DISCLOSURE The authors have no conflicts of interest or funding to disclose.

\section{REFERENCES}

1. Friedman AA, Letai A, Fisher DE, Flaherty KT. Precision medicine for cancer with next-generation functional diagnostics. Nat Rev Cancer. 2015;15:747-56.

2. Wan JCM, Massie C, Garcia-Corbacho J, et al. Liquid biopsies come of age: towards implementation of circulating tumour DNA. Nat Rev Cancer. 2017;17:223-38.

3. Heidrich I, Ackar L, Mossahebi Mohammadi P, Pantel K. Liquid biopsies: potential and challenges. Int $J$ Cancer. 2021;148:528-45.

4. Kilgour E, Rothwell DG, Brady G, Dive C. Liquid biopsy-based biomarkers of treatment response and resistance. Cancer Cell. 2020;37:485-95.

5. Leighl NB, Page RD, Raymond VM, et al. Clinical utility of comprehensive cell-free DNA analysis to identify genomic biomarkers in patients with newly diagnosed metastatic nonsmall cell lung cancer. Clin Cancer Res. 2019;25:4691-700.

6. Siravegna G, Mussolin B, Buscarino M, et al. Clonal evolution and resistance to EGFR blockade in the blood of colorectal cancer patients. Nat Med. 2015;21:795-801.

7. Parikh AR, Leshchiner I, Elagina L, et al. Liquid versus tissue biopsy for detecting acquired resistance and tumor heterogeneity in gastrointestinal cancers. Nat Med. 2019;25:1415-21.

8. Schwaederle M, Husain H, Fanta PT, et al. Use of liquid biopsies in clinical oncology: pilot experience in 168 patients. Clin Cancer Res. 2016;22:5497-505.

9. Rolfo C, Cardona AF, Cristofanilli M, et al. Challenges and opportunities of cfDNA analysis implementation in clinical practice: perspective of the International Society of Liquid Biopsy (ISLB). Crit Rev Oncol Hematol. 2020;151:102978.

10. Nakamura $\mathrm{Y}$, Taniguchi $\mathrm{H}$, Ikeda $\mathrm{M}$, et al. Clinical utility of circulating tumor DNA sequencing in advanced gastrointestinal cancer: SCRUM-Japan GI-SCREEN and GOZILA studies. Nat Med. 2020;26:1859-64.

11. Matsudera S, Kano Y, Aoyagi Y, et al. A pilot study analyzing the clinical utility of comprehensive genomic profiling using 
plasma cell-free DNA for solid tumor patients in Japan (PROFILE study). Ann Surg Oncol. 2021. https://doi.org/10.1245/ s10434-021-09856-5.

12. Razavi P, Li BT, Brown DN, et al. High-intensity sequencing reveals the sources of plasma circulating cell-free DNA variants. Nat Med. 2019;25:1928-37.

13. Bettegowda C, Sausen M, Leary RJ, et al. Detection of circulating tumor DNA in early- and late-stage human malignancies. Sci Transl Med. 2014;6:224ra224.
14. Zhang Q, Luo J, Wu S, et al. Prognostic and predictive impact of circulating tumor DNA in patients with advanced cancers treated with immune checkpoint blockade. Cancer Discov. 2020;10:1842-53.

Publisher's Note Springer Nature remains neutral with regard to jurisdictional claims in published maps and institutional affiliations. 